\title{
O Balanceamento de Réplicas em um Cluster HDFS com base na Confiabilidade dos Racks
}

\author{
Rhauani Weber Aita Fazul ${ }^{1}$, Paulo Vinicius Cardoso ${ }^{2}$, Patrícia Pitthan Barcelos ${ }^{2}$ \\ ${ }^{1}$ Laboratório de Sistemas de Computação (LSC) \\ ${ }^{2}$ Pós-Graduação em Ciência da Computação (PGCC) \\ Universidade Federal de Santa Maria (UFSM) \\ Santa Maria, Brasil \\ \{rwfazul, pcardoso, pitthan\}@inf.ufsm.br
}

\begin{abstract}
Resumo-A replicação de dados é um dos principais mecanismos de tolerância a falhas utilizados pelo HDFS. Porém, a forma de posicionamento das réplicas entre os nodos computacionais afeta diretamente o balanceamento e o desempenho do sistema. $O$ HDFS Balancer é uma solução disponibilizada pelo Apache Hadoop que visa equilibrar a distribuição dos dados. Todavia, sua política de operação atual não permite endereçar demandas de disponibilidade e confiabilidade ao redistribuir as réplicas entre os racks do cluster. Esse trabalho apresenta uma estratégia de balanceamento customizada para o HDFS Balancer baseada em fatores de confiança, que são calculados para cada rack a partir da taxa de falhas de seus nodos. Após detalhar a implementação, conduziu-se uma investigação experimental que permitiu validar e demonstrar a efetividade da estratégia desenvolvida.

Index Terms-replicação, balanceamento, confiança dos racks
\end{abstract}

\section{INTRODUÇÃO}

O Apache Hadoop [1] é uma plataforma de propósito geral dedicada ao armazenamento e ao processamento de dados em larga escala. Por oferecer um robusto sistema de gerência de dados e aplicações em arquiteturas de alto desempenho, tais como clusters e grids, o Hadoop é muito utilizado por empresas e pesquisadores. A simplicidade de configuração e de uso do framework, em conjunto com uma estrutura de desenvolvimento open-source, fazem do Hadoop uma poderosa ferramenta para análise e gerência de big data [2].

O ecossistema do Hadoop é composto por diversos componentes e ferramentas, capazes de fornecer alta escalabilidade e disponibilidade em ambientes distribuídos. Entre seus módulos primários estão o modelo de programação paralelo MapReduce, o gerenciador de recursos de propósito geral YARN e o sistema de arquivos distribuído HDFS [1]. Sendo a camada de armazenamento do Hadoop, o HDFS permite que inúmeras aplicações - coordenadas pelo YARN - manipulem dados simultaneamente de forma rápida e segura.

Para tal, no HDFS são implementados diversos mecanismos que buscam assegurar a confiabilidade e a disponibilidade do sistema mesmo no advento de falhas. Um dos principais mecanismos de tolerância a falhas adotados pelo HDFS é a replicação de dados. A replicação visa proporcionar confiabilidade por meio de redundância, além de estimular possíveis

Trabalho apoiado pelo Programa de Educação Tutorial (PET/MEC). melhorias de desempenho ao permitir que as aplicações explorem uma maior disponibilidade dos dados. Todavia, a forma de posicionamento das réplicas entre os nodos afeta diretamente o balanceamento e a localidade dos dados no cluster [3].

O HDFS Balancer [4] é uma solução nativa disponibilizada pelo Hadoop dedicada ao balanceamento de réplicas. Sua política de operação atual, entretanto, não permite que métricas e características específicas do cluster e dos racks que agrupam os nodos sejam levadas em consideração durante a redistribuição dos dados, de forma que demandas de disponibilidade e confiabilidade podem deixar de ser atendidas.

Este trabalho apresenta uma estratégia que customiza a política de balanceamento atual do HDFS Balancer com base na confiabilidade dos racks do HDFS. Desse modo, racks com uma maior confiabilidade são prioritários ao recebimento de um maior volume de dados durante a movimentação das réplicas. Para tal, para cada rack do cluster, calcula-se um fator de confiança através da taxa de falha de seus nodos. Com isso, após o balanceamento, racks com alta proporção de nodos falhos tendem a manter uma menor quantidade de dados em seus dispositivos de armazenamento.

O artigo está organizado em 7 seções. A Seção II apresenta o HDFS, aprofundando-se no mecanismo de replicação de dados. A Seção III é dedicada às causas e aos problemas do desbalanceamento de réplicas. A Seção IV relata os principais trabalhos relacionados. A Seção V descreve a implementação da solução proposta. A Seção VI exibe e discute os resultados obtidos. Por fim, a Seção VII apresenta as considerações finais e direciona os trabalhos futuros.

\section{Hadoop Distributed File System (HDFS)}

Dentro de um cluster HDFS segue-se uma arquitetura mestre-escravo formada por um NameNode (NN) e múltiplos DataNodes (DNs) [1]. O NN é o servidor mestre que gerencia o namespace e os metadados do sistema, controlando o acesso e a distribuição dos arquivos. Enquanto isso, os DNs são os workers que realizam efetivamente a recuperação e o armazenamento dos dados. Múltiplas instâncias de DNs possibilitam que a distribuição de dados ocorra em diferentes máquinas do cluster, sendo estas conectadas por rede.

Desde sua criação, o HDFS foi otimizado para armazenar dados na escala de petabytes [4]. Para lidar com esse volume 
de dados massivo, o HDFS define uma estrutura de armazenamento própria, onde, quando um arquivo é inserido no sistema, ao invés de salvá-lo em sua forma original, gera-se uma sequência de blocos. Os blocos consistem em segmentos de dados criados de forma automática a partir do particionamento do arquivo inicial. Todos os blocos, com exceção do último que pode ser menor, possuem um tamanho fixo: $128 \mathrm{MB}$ por padrão, a partir da segunda versão do Hadoop [2].

A alta escalabilidade do Hadoop torna possível que os clusters possuam milhares de nodos trabalhando em conjunto, responsáveis tanto por computação como por armazenamento de dados. Mesmo que alguns componentes de hardware falhem, é importante que o sistema mantenha sua consistência, de modo que blocos comprometidos possam ser restaurados. Para tal, meios que garantam a integridade e a disponibilidade dos dados através da detecção e recuperação mediante falhas são imprescindíveis. O mecanismo de tolerância a falhas mais expressivo no HDFS é a replicação de dados.

\section{A. Replicação de Dados}

Atuando como um importante mecanismo de tolerância a falhas (TF) e como uma estratégia para suprir altas demandas de disponibilidade e acesso aos dados, o HDFS implementa a replicação de blocos. A replicação baseia-se na criação de cópias dos blocos de dados presentes no sistema (réplicas), de modo a aumentar a confiabilidade e a disponibilidade de dados com base em redundância.

Os blocos replicados são armazenados em diferentes nodos do cluster. Assim, caso um nodo falhe, seus blocos podem ser acessados a partir de um ou mais DNs que mantenham suas réplicas. $\mathrm{O}$ número de réplicas a ser gerado para cada bloco é definido por arquivo a partir de um parâmetro configurável, o Fator de Replicação (FR). Uma aplicação pode especificar o FR no momento da criação de um arquivo, sendo possível modificá-lo posteriormente [1].

A replicação garante que, com um FR de $n$, os dados armazenados no HDFS fiquem seguros mesmo na ocorrência de $n-1$ falhas simultâneas. Todavia, a replicação inicial dos blocos no momento da escrita de um arquivo no HDFS não é suficiente para garantir TF. Ao executar sobre hardware comum, o HDFS está sujeito a falhas frequentes. Para proteger e manter a integridade dos dados em cenários onde ocorram falhas consecutivas em um curto intervalo de tempo, o $\mathrm{NN}$ precisa monitorar e controlar ativamente o número de réplicas disponíveis e não corrompidas de cada bloco e, quando aplicável, realizar a re-replicação dos dados [2].

Para todos os blocos que necessitem de re-replicação, o NN deve agir e reiniciar o processo de replicação em outros DNs, assim mantendo a conformidade com o FR definido. Para tal, o NN seleciona um DN que contenha a cópia do bloco a ser re-replicado e um DN como destino para a nova réplica. Esta escolha é feita de forma arbitrária e, assim como a replicação, é realizada de forma transparente pelo HDFS. O processo de re-replicação, dentre outros motivos, pode ser originado devido a falhas no funcionamento de algum DN [1].
Falhas na comunicação entre o NN e o DN são identificadas pela ausência de mensagens heartbeat: um mecanismo de TF que permite detectar falhas operacionais em DNs [2]. Periodicamente, os DNs enviam heartbeats ao NN a fim de notificálo sobre seu estado de funcionamento. Caso o NN não receba heartbeats de um DN dentro de um determinado intervalo de tempo, o NN marca o DN em questão como inativo. Além de não receber novas requisições de entrada e saída (E/S), o FR dos blocos de um DN inativo é decrementado. Se o número de réplicas de um bloco for inferior ao FR especificado, a re-replicação pode ser disparada pelo NN a partir de cópias sobressalentes armazenadas em algum dos DNs ativos. Desse modo, a conformidade com o FR é restaurada, preservando a disponibilidade dos dados caso novas falhas ocorram.

Ao realizar a replicação e a re-replicação, uma quantidade considerável de blocos deve ser distribuída entre os DNs. O posicionamento desses blocos é importante para assegurar a disponibilidade e o desempenho do sistema. Atuando como o nodo mestre, o NN é encarregado das decisões acerca da replicação dos blocos no HDFS. Para aumentar a disponibilidade e garantir uma melhor comunicação entre as réplicas e os clientes, o NN segue uma política de posicionamento como referência para a distribuição dos dados no cluster.

\section{B. Política de Posicionamento de Réplicas (PPR)}

Instâncias do HDFS, em geral, são compostas por múltiplos nodos, dispostos em diferentes racks. Sendo um sistema que preza pela tolerância a falhas, o HDFS deve evitar a perda de dados mesmo que um rack inteiro falhe. Ao ser armazenado, um bloco é replicado com base no FR de seu arquivo. Para cada bloco a ser armazenado no HDFS, o NN precisa selecionar os DNs para o recebimento de suas réplicas. Esta escolha deve ser realizada visando manter a disponibilidade dos blocos em caso de falhas e aprimorar o desempenho do sistema em operações sobre os dados armazenados.

Para tal, o NN guia-se por um modelo inteligente para o posicionamento das réplicas, otimizado para aumentar a disponibilidade e o desempenho do HDFS de acordo com a arquitetura do cluster [2]. O modelo corrente, conhecido como Política de Posicionamento de Réplicas (PPR) [1], é aplicado da seguinte forma (Apache Hadoop versão 2): (i) a primeira réplica é armazenada no mesmo nodo do cliente, porém se o cliente HDFS estiver executando fora do cluster, um DN é escolhido aleatoriamente pelo NN (embora o sistema evite, na medida do possível, escolher nodos que estejam com alto tráfego de comunicação); (ii) as duas réplicas seguintes são armazenadas em diferentes DNs de um mesmo rack remoto, sendo este diferente do rack da primeira réplica; (iii) caso o FR seja maior que o padrão (três réplicas por bloco), as réplicas seguintes são posicionadas de forma arbitrária, porém mantendo o número de réplicas por rack abaixo do valor limite resultante de $(($ réplicas -1$) /$ racks $)+2$.

Em relação à sobrecarga no processo de escrita, que envolve o armazenamento das múltiplas réplicas de um mesmo bloco, o HDFS aplica, quando possível, a técnica de pipeline de replicação [2]. A partir da definição dos DNs para o rece- 
bimento das réplicas, estabelece-se um pipeline onde DNs podem, simultaneamente, receber e encaminhar dados. Além de aprimorar a operação de escrita, o pipeline permite que todo processo de replicação seja transparente ao usuário, que apenas precisa interagir com um único nodo.

A replicação também possibilita otimizar operações voltadas à leitura e à recuperação dos dados, onde o HDFS tira proveito da redundância espacial dos blocos para suprir altas demandas de acesso. Como o HDFS segue uma estratégia rack-awareness, é possível identificar o rack mais próximo do cliente que possua a réplica requisitada, diminuindo o tempo gasto com o tráfego de dados [1].

Desta forma, a PPR garante um bom equilíbrio em relação [2]: (i) à TF e à disponibilidade dos dados mesmo em caso de falha de um rack inteiro; (ii) ao desempenho na escrita dos blocos, já que, na maioria dos casos, a largura de banda entre nodos de um mesmo rack é maior do que a largura de banda inter-rack e a PPR, ao armazenar mais de uma réplica em um mesmo rack, faz com que os dados trafeguem por um menor número de comutadores (network switches); e (iii) ao desempenho na leitura dos blocos devido a maior disponibilidade dos dados (operações de leitura podem ser realizadas utilizando a largura de banda de racks distintos), assim tornando o acesso aos dados mais rápido.

Embora aumente a disponibilidade do sistema em caso de falhas (redundância de dados em racks distintos) e contribua com uma melhor utilização dos recursos computacionais do cluster (operações de E/S podem tirar proveito da largura de banda de múltiplos racks), a PPR não distribui os blocos de forma igualitária entre os DNs [1].

\section{BALANCEAMENTO DE RÉPliCAS}

O HDFS foi projetado para operar sobre um volume massivo de dados. $\mathrm{O}$ armazenamento de arquivos maiores resulta em uma maior quantidade de blocos, os quais devem ser posicionados pelo NN. A PPR garante um balanceamento mínimo (réplicas de um mesmo bloco não recaem em um mesmo DN), porém não é suficiente para manter o cluster balanceado [3].

A PPR pode favorecer o desbalanceamento de réplicas em dois sentidos. Ao selecionar um rack para manter $2 / 3$ das réplicas de um determinado bloco (considerando o FR padrão), tende-se a favorecer o desequilíbrio inter-rack. Já o desbalanceamento intra-rack (inter-DN) é resultante da arbitrariedade na escolha dos nodos. Em geral, qualquer DN que satisfaça as restrições impostas pela PPR é eletivo ao armazenamento da réplica, sendo a decisão final realizada pelo $\mathrm{NN}$ [2].

Além disso, outros aspectos podem contribuir com o desbalanceamento de réplicas no HDFS, tais como: (i) a adição de um novo nodo ao cluster, já que este irá competir igualmente com outros DNs para o recebimento dos blocos replicados, resultando em um período de subutilização significativo [4]; (ii) o processo de re-replicação, que está sujeito à mesma política da replicação inicial; e (iii) o comportamento da aplicação do cliente que, caso execute diretamente em um DN do cluster, faz com que este, de acordo com a PPR, armazene sempre uma das réplicas localmente.
Sendo um sistema baseado no modelo de acesso WORM (write once, ready many), muitos dos esforços do HDFS são voltados a maximizar a vazão durante operações de leitura dos dados [2]. O posicionamento dos blocos no cluster é um fator crítico para a disponibilidade dos dados e para o desempenho de aplicações de E/S [4]. Um bom posicionamento das réplicas permite reduzir tráfego de dados entre switches, o que pode se tornar um gargalo em sistemas de computação intensiva [5].

Um dos princípios do Hadoop é mover a aplicação até os dados, evitando mover os dados propriamente ditos [1]. Trazer a computação para perto dos dados, base do processamento do Hadoop, é conhecido como localidade dos dados (data locality) [2]. Esta funcionalidade permite aprimorar a eficiência da plataforma no processamento de grandes datasets, já que, por ser local, o acesso aos blocos torna-se mais rápido e menos custoso em termos de consumo de banda.

Como cada bloco é replicado, por padrão, em três DNs diferentes, a probabilidade de que uma tarefa map consiga processar a maioria dos blocos localmente é alta [5]. Entretanto, uma distribuição de réplicas desequilibrada tende a afetar a localidade das tarefas $\operatorname{map}^{1}$ do MapReduce [2], podendo ocasionar um maior número de transferências intrarack ou off-rack e, assim, consumindo a largura de banda do cluster. Além disso, o desbalanceamento pode acarretar em sobrecarga para os DNs com maior utilização do cluster (nodos com mais blocos armazenados), prejudicando ainda mais o desempenho na execução de aplicações I/O bound.

\section{TRABALHOS RELACIONADOS}

Estudos passados analisaram a influência da replicação e da re-replicação de blocos no balanceamento de réplicas do HDFS [6]. Ao atestar que o desequilíbrio na distribuição dos dados é uma realidade presente no sistema de arquivos do Hadoop, conduziu-se uma investigação experimental acerca da efetividade da PPR, evidenciando os requisitos necessários para um posicionamento de réplicas eficiente [3].

Uma forma de promover o balanceamento de réplicas no HDFS é agir no momento da distribuição inicial dos blocos. Com isso, é possível impedir - ou reduzir as chances - que o cluster fique desequilibrado. Muitas das soluções existentes na literatura [7] [8], envolvem a criação de novas políticas de posicionamento de réplicas que, proativamente, de forma direta ou indireta, contribuem com o balanceamento.

Outra abordagem para endereçar o balanceamento é através de estratégias reativas, onde disparam-se ações corretivas a fim de tornar o posicionamento dos dados entre os nodos mais homogêneo. Para tal, os blocos já armazenados no sistema de arquivos são redistribuídos dentro do cluster, visando promover o balanceamento inter-rack e/ou entre DNs.

Exemplos dessa abordagem incluem [9], onde é proposto um algoritmo aprimorado para o balanceamento entre racks com base em prioridade. A estratégia atua primariamente em

\footnotetext{
${ }^{1}$ As tarefas reduce não tiram vantagem da localidade dos dados [2]. Em geral, a entrada de uma tarefa de redução é a saída de todas as tarefas de mapeamento previamente processadas, que são transferidas, em totalidade, para o local onde a função de redução definida pelo usuário é executada.
} 
equilibrar racks sobrecarregados, com isso reduzindo as chances de falha total de rack devido à sobrecarga e contribuindo com uma distribuição mais uniforme dos dados. Em [8], é introduzido um algoritmo modificado (LatencyBalancer) como parte do balanceador Tula que, além da utilização dos DNs, considera variações na latência de escrita e leitura dos discos de armazenamento dos nodos para a realocação dos dados no HDFS. Com isso, os DNs que apresentarem menor latência de disco recebem um número maior de blocos. Os resultados com esta estratégia demostraram reduções de cerca de $20 \%$ no tempo de execução de aplicações MapReduce.

Sabendo que o processo de balanceamento pode ser trabalhoso em função do estado em que o cluster se encontra, em [10] otimiza-se a redistribuição das réplicas a partir de diferenças de hardware dos nodos. Em contraste com o balanceador Tula, que não considera variações nos recursos de processamento e memória dos nodos, o algoritmo de balanceamento de [10] baseia-se na capacidade de computação dos DNs. Sendo voltado a instâncias do Hadoop executando em ambientes heterogêneos, os blocos são redistribuídos apenas para DNs específicos, determinados a partir de uma classificação inicial pela heterogeneidade e desempenho de cada nodo. Esta restrição é utilizada como uma estratégia para reduzir o tempo gasto com a transferência dos dados.

Outra solução reativa para o balanceamento de réplicas é o HDFS Balancer [4]: ferramenta responsável pela análise do posicionamento dos blocos armazenados no sistema de arquivos para posterior redistribuição de dados entre os DNs. Por ser a base para o desenvolvimento deste trabalho, a Seção IV-A detalha o funcionamento do balanceador do HDFS.

\section{A. HDFS Balancer}

O HDFS Balancer é uma ferramenta integrada na distribuição do Hadoop que visa o balanceamento de réplicas entre os dispositivos de armazenamento do HDFS. A partir de sua política de execução padrão, o balanceador opera iterativamente movimentando blocos de DNs que apresentarem uma alta utilização (origem) para DNs que possuírem um menor volume de dados armazenado (destino) [11]. Sua execução é disparada sob demanda pelo administrador do cluster.

Durante a execução do balanceador, os dispositivos de armazenamento dos DNs são divididos em grupos de acordo com seus tipos (e.g. disco rígido e SSD). Sendo $i$ um DN qualquer e $t$ um tipo de dispositivo de armazenamento, considera-se: (i) $G_{i, t}$ como o grupo de dispositivos de armazenamento do tipo $t$ do $\mathrm{DN} i$; (ii) $U_{i, t}$ como a porcentagem representando a utilização do grupo dos dispositivos do tipo $t$ do DN $i$; e (iii) $U_{\mu, t}$ como a porcentagem representando a média de utilização de todos os dispositivos do tipo $t$ do cluster.

A operação de balanceamento é guiada por um threshold, que é passado como parâmetro para a execução do balanceador. Representado como uma porcentagem no intervalo de $0 \%$ a $100 \%$, o threshold limita a diferença máxima que a $U_{i, t}$ de um $G_{i, t}$ e a utilização geral do cluster $\left(U_{\mu, t}\right)$ pode assumir [2]. Quando a utilização de cada grupo estiver dentro desse limite, o cluster é tido como balanceado.
Por exemplo, considerando o threshold padrão de $10 \%$ e, supondo que o cluster esteja com metade de sua capacidade ocupada $\left(U_{\mu, t}\right.$ em $50 \%$ ), a ferramenta irá executar até que todos os dispositivos de armazenamento de todos os DNs estejam com utilização entre $40 \%$ e $60 \%$. Ao reduzir o threshold aumenta-se o equilíbrio do cluster, todavia maior será o esforço - em termos de processamento e de transferência de dados - necessário para realizar o balanceamento.

Uma iteração de balanceamento parte da inicialização de um dispatcher para a movimentação dos blocos e da obtenção de todos os DataNodeStorageReports dos DNs ativos do cluster. A partir disto, três ações principais são realizadas [11]: (i) classificação dos $G_{i, t}$ dos DNs, efetuada pelo método $\mathrm{Bal}$ ancerinit; (ii) pareamento dos grupos origem-destino, exercido pelo método chooseStorageGroups; e (iii) criação de uma thread responsável por decidir os blocos a serem movimentados e efetivar a transferência através de um método do dispatcher.

O método Balancer.init estabelece, para cada $G_{i, t}$, o número máximo de bytes que pode ser movimentado na iteração. A partir disto, realizam-se os cálculos necessários para a definição da variável maxSize2Move, que equivale ao volume de dados (em bytes) necessário para levar a $U_{i, t}$ até a $U_{\mu, t}$.

Em seguida, cada $G_{i, t}$ é classificado em [11]: (i) superutilizado $\left(U_{i, t}>U_{\mu, t}+\right.$ threshold); (ii) acima da média $\left(U_{\mu, t}+\right.$ threshold $\left.\geq U_{i, t}>U_{\mu, t}\right)$; (iii) abaixo da média $\left(U_{\mu, t} \geq U_{i, t} \geq U_{\mu, t}-\right.$ threshold); ou (iv) subutilizado $\left(U_{\mu, t}\right.$ - threshold $>U_{i, t}$ ). Os grupos são então adicionados em listas globais de acordo com as suas classificações.

Após, o método chooseStorageGroups determina todos os nodos que irão participar da iteração. Cada $G_{i, t}$ superutilizado (origem) é pareado com um ou mais $G_{i, t}$ subutilizados (destino) em uma relação $1-N$. Se algum grupo superutilizado possuir maxSize2Move satisfeito, ele é removido da lista e não será mais pareado na iteração corrente. Para os grupos superutilizados remanescentes, são selecionados candidatos nos $G_{i, t}$ classificados como abaixo da média (destino). Se ainda houver algum $G_{i, t}$ subutilizado, procuram-se candidatos entre os $G_{i, t}$ acima da média restantes (origem).

Com o pareamento finalizado, o método dispatchAndCheckContinue inicia uma thread para a seleção e a transferência dos blocos entre os grupos. Quando as movimentações forem concluídas, a iteração é encerrada e o resultado preliminar do balanceamento é registrado. Por fim, todas as listas e mapeamentos são resetados para as iterações futuras, que serão executadas caso o cluster ainda não esteja balanceado.

\section{Política de Balanceamento Customizada}

A operação do HDFS Balancer é guiada por uma política de balanceamento, porém, por operar de forma generalizada, a política atual nem sempre é satisfatória e otimizada para os diferentes contextos e cenários de uso em que o HDFS é empregado. Através da compreensão do algoritmo de funcionamento da ferramenta e da revisão do estado da arte em balanceamento de dados em sistemas distribuídos, foi elencado um conjunto de possíveis otimizações e novas funcionalidades para o balanceador nativo do HDFS [12]. 
As modificações propostas foram estruturadas na forma de uma política de balanceamento de réplicas customizada, que faz uso de um sistema de prioridades para realizar a redistribuição dos blocos com base na topologia do cluster e em diferentes métricas de usabilidade do sistema. Com isso, permite-se que o balanceamento atenda demandas de uso específicas de aplicações reais, seja através de otimizações na execução do Hadoop em ambientes heterogêneos ou em explorar potenciais ganhos de confiabilidade e disponibilidade durante a movimentação dos dados.

As prioridades da política customizada foram agrupadas em categorias de acordo com suas características de funcionamento, conforme mostra a Tabela [1] [13]. A categoria "capacidade dos nodos" realiza a priorização com base em diferenças de hardware dos DNs, enquanto a categoria "distribuição dos dados" visa o aumento da disponibilidade dos blocos. Já as prioridades da categoria "estado dos nodos", fazem uso de métricas recuperadas em tempo de execução para reduzir o overhead ocasionado pelo processo de balanceamento.

Tabela I

SISTEMA DE PRIORIDADES IMPLEMENTADO PELA POLÍTICA DE BALANCEAMENTO DE RÉPLICAS CUSTOMIZADA.

\begin{tabular}{c|c}
\hline Categoria & Prioridade \\
\hline Capacidade dos nodos & $\begin{array}{c}\text { Capacidade de armazenamento } \\
\text { Capacidade de processamento }\end{array}$ \\
\hline Distribuição dos dados & Disponibilidade dos dados \\
\hline Estado dos nodos & Utilização dos nodos \\
& Classificação dos nodos \\
Estado dos racks & Confiabilidade dos racks \\
& Utilização dos racks \\
\hline
\end{tabular}

Este trabalho enfoca uma das prioridades da categoria "estado dos racks", denominada confiabilidade dos racks, que visa customizar a política padrão do HDFS Balancer com base em características dos racks que agrupam os DNs do HDFS. Na Seção V-A a prioridade em questão é detalhada.

\section{A. Confiabilidade dos Racks}

A prioridade de confiabilidade dos racks avalia os racks do sistema com base na suscetibilidade a falhas de seus DNs. Assim, antes de selecionar um rack para o recebimento dos blocos, realiza-se uma verificação da quantidade de DNs inativos no cluster, esforçando-se em armazenar uma maior quantidade de dados em racks com uma menor ocorrência de falhas. Embora DNs inativos sejam resultado tanto de falhas de hardware quanto de software, racks que possuírem uma proporção elevada entre DNs inativos em relação aos ativos podem estar passando por um período de sobrecarga.

Na política padrão do balanceador apenas os DNs ativos são levados em consideração, porém para a implementação dessa prioridade os DNs inativos também precisam ser considerados. Dessa forma, foi adicionado o método getDeadDatanodeStorageReport à classe NameNodeConnector, que retorna a lista dos reports dos DNs inativos do cluster. Esse método é similar ao método getLiveDatanodeStorageReport (já existente no código original do HDFS Balancer), diferindo apenas no valor da enumeração DatanodeReportType, que é repassado ao método getDatanodeStorageReport da classe ClientProtocol (o método padrão utiliza a enumeração com o valor " $L I V E$ ", o novo método utiliza o valor " $D E A D$ ”).

Após, adicionou-se um método na classe Dispatcher dedicado à coleta dos reports de modo similar ao processo já realizado pelo método Dispatcherinit, porém consumindo a lista dos DNs inativos ao invés dos ativos. A modificação mais expressiva, por sua vez, se deu no arquivo principal Balancer.

Inicialmente, implementou-se um método destinado ao controle e ao mapeamento da quantidade de DNs (ativos e inativos) do cluster e seus respectivos racks. A Figura 1 exibe o algoritmo do método mapRacksAndDatanodes, onde são preenchidas as estruturas liveDatanodeMap (L. 2 a 9) e deadDatanodeMap (L. 10 a 17). Estas estruturas são consumidas pelo método computeRFactor, responsável por quantificar a confiabilidade dos racks de acordo com a suscetibilidade a falhas de seus DNs. A chamada desse método foi incorporada em um momento anterior à chamada do método Balancerinit.

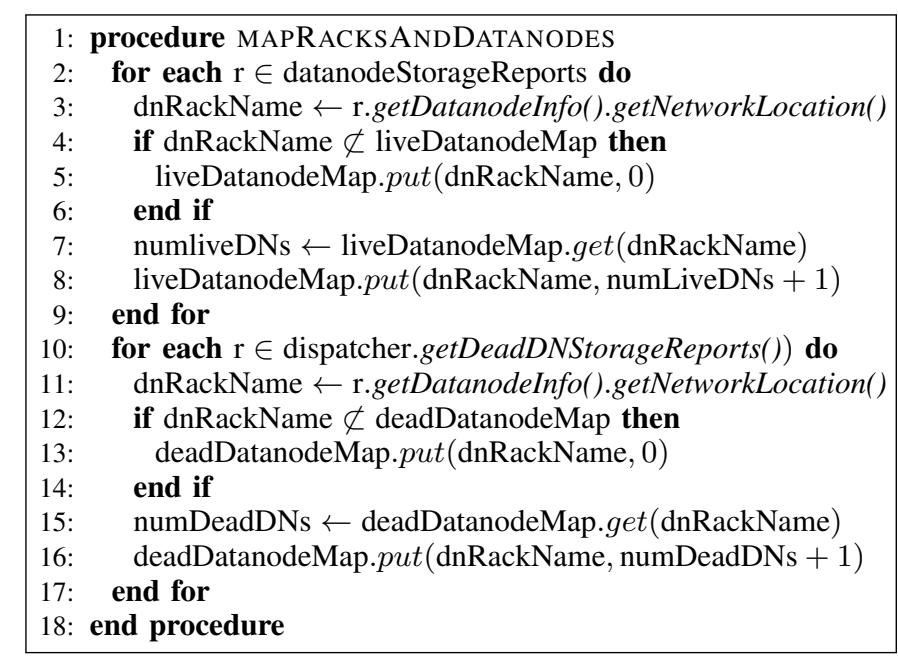

Figura 1. Método para mapeamento dos DNs ativos e inativos em cada rack.

A Figura 2 exibe o algoritmo definido no método computeRFactor. Inicialmente, é realizado o cálculo da taxa de falhas de cada um dos racks (L. 2 a 10). Se um rack não possuir DNs inativos, sua taxa de falhas será zero. Caso contrário, calcula-se o quanto estes representam em relação ao total de DNs do rack (L. 7). Em seguida, os valores calculados para cada um dos racks do cluster (intervalo de 0 a 1) são inseridos na estrutura failureRateMap (L. 9).

Após, estima-se um fator de confiança para cada rack (rFactor). Para tal, aplicou-se a normalização min-max nas taxas de falhas dos racks (L. 16), assim permitindo a definição de um intervalo controlado de valores. Com a normalização, torna-se o valor mínimo de um conjunto de valores em 0 , o valor máximo em 1 e, qualquer outro valor, em um decimal proporcional entre 0 e 1 . A Equação 1 aplica a normalização 
: procedure COMPUTERFACTOR

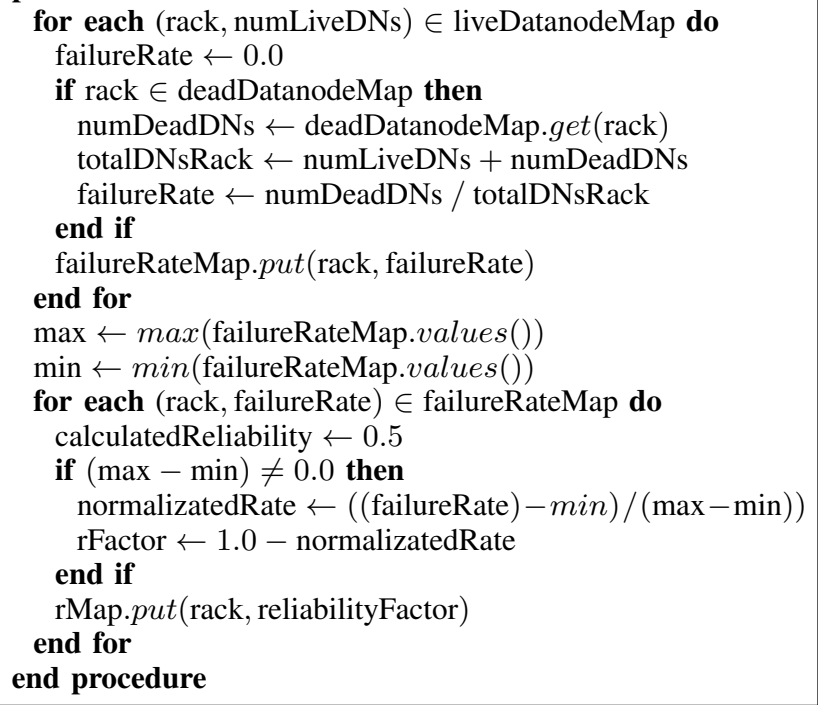

Figura 2. Método responsável por quantificar a confiabilidade dos racks.

min-max sobre a taxa de falhas de um rack $r\left(T_{r}\right)$ a fim de obter seu respectivo fator de confiança, i.e. rFactor $\left(T_{r}^{\prime}\right)$.

$$
T_{r}^{\prime}=\frac{T_{r}-\min }{\max -\min }
$$

Caso não haja ocorrência de DNs falhos nos racks do cluster, max terá valor zero e, assim, $T_{r}^{\prime}$ passa a ser um valor médio de 0,5 (L. 14), fazendo com que a definição de maxSize2Move para os grupos de dispositivos de armazenamento seja similar ao cálculo realizado pela política padrão do HDFS Balancer $\left(\left|U_{i, t}-U_{\mu, t}\right|\right)$. Caso contrário, o fator de confiança é definido com base na quantidade de DNs ativos e inativos no rack (L. 17). Vale observar que, caso o valor armazenado em failureRateMap para um determinado rack seja zero, $T_{r}^{\prime}$ terá o valor 1 (motivo do uso do valor inverso na linha 17).

Os fatores de confiança dos racks são salvos na estrutura rMap (L. 19), que será posteriormente acessada pelo método calcMaxSize2MoveBasedOnRFactor. Este método, cujo algoritmo é exibido na Figura 3, estabelece o valor de maxSize2Move de um $G_{i, t}$ de acordo com o fator de confiança de seu rack. Sua chamada foi incorporada ao método Balancer.init em um momento anterior à classificação dos grupos.

Inicialmente, para cada $G_{i, t}$, calcula-se a diferença de sua utilização $\left(U_{i, t}\right)$ para o limite superior $\left(U_{\mu, t}+\right.$ threshold $)$ e para o limite inferior $\left(U_{\mu, t}-\right.$ threshold $)$ que são considerados pelo balanceador (L. 4 e 5). Esses valores, por sua vez, permitem definir a quantidade de bytes necessária para levar a $U_{i, t}$ de um grupo até o limite máximo para que este seja considerado como acima ou abaixo da média (L. 6 e 7). Em seguida, o valor de maxSize2Move é definido de acordo com a classificação e a $T_{r}^{\prime}$ do rack ao qual o $G_{i, t}$ pertence.

De forma geral, o valor de maxSize2Move para um $G_{i, t}$ de um rack com uma $T_{r}^{\prime}$ elevada, aproxima-se do volume de bytes necessário para elevar sua $U_{i, t}$ até o limite superior. Já para um $G_{i, t}$ de um rack com uma $T_{r}^{\prime}$ baixa, aproxima-se do

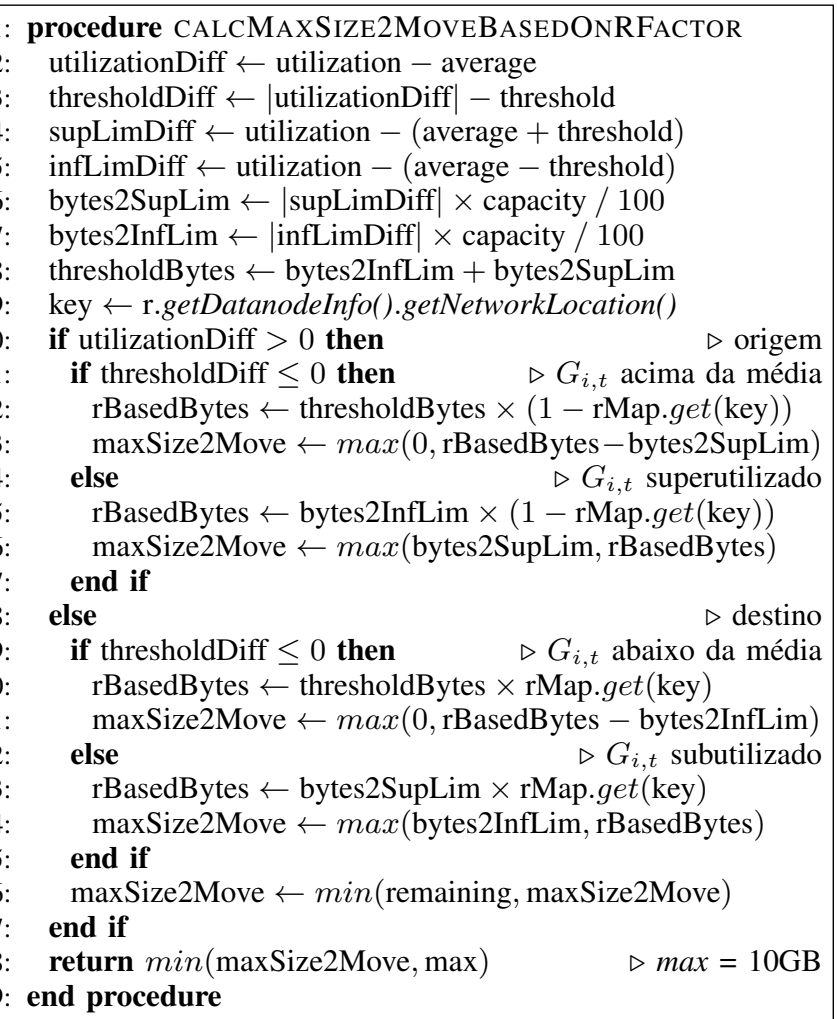

Figura 3. Método dedicado ao cálculo customizado da variável maxSize2Move de um $G_{i, t}$ com base no fator de confiança de seu rack $\left(T_{r}^{\prime}\right)$.

volume de bytes necessário para reduzir sua $U_{i, t}$ até o limite inferior. Para idealizar este comportamento, é importante observar que, em um grupo origem (superutilizado ou acima da média), maxSize2Move determina a redução máxima de sua $U_{i, t}$ (i.e. não é possível aumentar sua utilização para um valor superior à $U_{i, t}$ atual). Analogamente, para um grupo destino (subutilizado ou abaixo da média), maxSize2Move determina a extensão máxima de sua $U_{i, t}$ (i.e. não é possível reduzir sua utilização para um valor inferior à $U_{i, t}$ atual).

Sendo assim, se a $T_{r}^{\prime}$ do rack de um $G_{i, t}$ classificado como acima da média resultar em uma utilização superior à $U_{i, t}$ atual do grupo, maxSize2Move deverá ter o valor zero (L. 13), garantindo dessa forma que sua utilização não seja reduzida. Similarmente, se a $T_{r}^{\prime}$ do rack de um $G_{i, t}$ abaixo da média resultar em uma utilização inferior à $U_{i, t}$ atual do grupo, maxSize2Move também deverá ter o valor zero (L. 21), garantindo que sua utilização não seja aumentada.

Além disso, em $G_{i, t}$ superutilizados de racks com alta $T_{r}^{\prime}$, o valor de maxSize2Move deve, ao mínimo, ser o suficiente para permitir a classificação do grupo como acima da média (garantido pelo método $\max$ na linha 16). Já em $G_{i, t}$ subutilizados de racks com baixa $T_{r}^{\prime}$, o valor de maxSize2Move deve ao menos ser suficiente para a classificação do grupo como abaixo da média (garantido pelo método max na linha 24).

Por fim, são aplicadas duas validações (definidas também no cálculo realizado pela política padrão do balanceador): (i) em grupos destino (utilizationDiff menor que zero), o 
valor da variável maxSize2Move deve ser limitado pelo espaço de armazenamento restante no $G_{i, t}$ (L. 26); e (ii) maxSize2Move deve respeitar o valor de max (dado pela propriedade $d f$ s.balancer.max-size-to-move) (L. 28).

\section{EXPERIMENTAÇÃO E DISCUSSÃO}

A prioridade de confiabilidade dos racks foi avaliada através de um experimento realizado na plataforma GRID'5000, com o Hadoop (versão 2.9.2) operando em modo totalmente distribuído, executando sobre uma distribuição Debian 9.9. Todos os nodos configurados pertenciam ao cluster suno, localizado no site Sophia, cada um com 2 processadores Intel Xeon E5520 (4 cores por CPU), 32GB de memória RAM, 508,54GB de armazenamento HDD e conexão Gigabit Ethernet.

A carga de dados foi realizada pelo TestDFS IO [2] (versão 1.8): um benchmark distribuído proprietário do Hadoop, que permite medir o desempenho do HDFS através da execução de tarefas MapReduce focadas em E/S intensiva. Foram escritos 10 arquivos de $30 \mathrm{~GB}$ cada um com $\mathrm{FR}$ padrão de 3 réplicas por bloco, totalizando aproximadamente 908,55GB de dados no sistema. Para que a suscetibilidade a falhas dos racks fosse quantificada pela prioridade, falhas de DN foram induzidas durante a escrita dos arquivos pelo benchmark.

O cenário de testes considerou 15 DNs dispostos em 3 racks distintos $\left(R_{1}, R_{2}, R_{3}\right)$, cada um mantendo 3,5 e 7 DNs. A introdução das falhas foi realizada pelo comando kill do Linux aos processos dos DNs selecionados. Ao total, foram induzidas 2 falhas de DN no rack $R_{2}$ e 4 falhas no rack $R_{3}$. Com isso, após as falhas, cada rack manteve exatamente 3 DNs ativos. Os DNs para indução das falhas foram escolhidos arbitrariamente.

As falhas são identificadas pelo $\mathrm{NN}$ em decorrência da ausência de mensagens heartbeat em um intervalo prédefinido. Como o tempo para o NN marcar um DN como inativo é relativamente longo, este período foi reduzido (através de um conjunto de parâmetros de configuração do HDFS) para aproximadamente 20 segundos. O tempo para a ocorrência da primeira falha de $\mathrm{DN}$ e o intervalo entre as falhas foi fixado em 60 segundos. Desta forma, o processo de re-replicação é disparado pelo NN e efetivamente concluído antes da escrita dos arquivos ser finalizada pelo TestDFSIO.

A capacidade de armazenamento total do sistema, considerando apenas os DNs ativos, era de 4,47TB ( $U_{\mu, D I S K}$ em 20,95\%). A Tabela II exibe a ocupação de cada DN ativo, em GB $\left(O_{i, D I S K}\right)$, e sua respectiva porcentagem de utilização $\left(U_{i, D I S K}\right)$. O fator de confiança de cada rack $\left(T_{r}^{\prime}\right)$ foi calculado de acordo com a normalização min-max baseada na quantidade de DNs inativos no rack no momento do balanceamento. Com isso, dada a quantidade de falhas de DN induzidas em cada rack, $T_{R 1}^{\prime}>T_{R 2}^{\prime}>T_{R 3}^{\prime}$.

A utilização total dos racks (proporção da ocupação acumulada para a capacidade total de seus DNs) antes do balanceamento era de, respectivamente, $58,76 \%, 60,66 \%$ e $59,24 \%$. Sendo assim, o rack com a maior $T_{r}^{\prime}\left(R_{1}\right)$ estava mantendo um volume de dados menor que os demais racks, os quais apresentavam incidência de falhas de DN ( $R_{2}$ com 2 DNs inativos e $R_{3}$ com 4 DNs inativos). Após o balanceamento
Tabela II

ESTADO DO HDFS ANTES E APÓS O BALANCEAMENTO DE RÉPLICAS COM A PRIORIDADE DE CONFIABILIDADE DOS RACKS.

\begin{tabular}{ccc|cc|cc}
\hline \multirow{2}{*}{ Rack } & \multirow{2}{*}{$\mathbf{T}_{\mathbf{r}}^{\prime}$} & \multirow{2}{*}{ DataNode } & \multicolumn{2}{|c|}{ s/ balanceamento } & \multicolumn{2}{c}{ c/ balanceamento } \\
\cline { 4 - 7 } & & $O_{i, D I S K}$ & $U_{i, D I S K}$ & $O_{i, D I S K}$ & $U_{i, D I S K}$ \\
& & & $(\mathrm{~GB})$ & $(\%)$ & $(\mathrm{GB})$ & $(\%)$ \\
\hline \multirow{3}{*}{$R_{1}$} & \multirow{2}{*}{1,0} & $\mathrm{DN}_{01}$ & 78,37 & 15,41 & 123,52 & 24,29 \\
& $\mathrm{DN}_{02}$ & 135,51 & 26,65 & 126,23 & 24,82 \\
& & $\mathrm{DN}_{03}$ & 84,92 & 16,70 & 126,01 & 24,78 \\
\hline \multirow{3}{*}{$R_{2}$} & \multirow{2}{*}{0,3} & $\mathrm{DN}_{04}$ & 96,16 & 18,91 & 95,75 & 18,83 \\
& & $\mathrm{DN}_{05}$ & 91,20 & 17,93 & 102,30 & 20,12 \\
& $\mathrm{DN}_{06}$ & 121,13 & 23,82 & 98,17 & 19,30 \\
\hline \multirow{2}{*}{$R_{3}$} & \multirow{2}{*}{0,0} & $\mathrm{DN}_{07}$ & 75,83 & 14,91 & 75,97 & 14,94 \\
& $\mathrm{DN}_{08}$ & 149,50 & 29,40 & 85,13 & 16,74 \\
& & $\mathrm{DN}_{09}$ & 75,93 & 14,93 & 75,97 & 14,94 \\
\hline
\end{tabular}

com base na confiança dos racks, a utilização de $R_{1}, R_{2}$ e $R_{3}$ foi levada para, respectivamente, $73,89 \%, 58,25 \%$ e $46,62 \%$. Sendo assim, observa-se que, estando de acordo com a confiabilidade calculada para cada rack do cluster (i.e. fatores de confiança), $U_{R 1, D I S K}>U_{R 2, D I S K}>U_{R 3, D I S K}$.

De forma a avaliar o impacto do balanceamento com a estratégia de priorização proposta no desempenho do sistema, foram realizadas 20 execuções distintas do TestDFSIO voltadas à leitura dos dados armazenados no HDFS. A análise por meio desse benchmark mostra-se interessante pois, através de seu comportamento característico $I / O$ bound, possibilita-se a investigação de possíveis melhorias na localidade espacial dos dados impulsionadas pelo equilíbrio das réplicas no cluster.

A Figura 4 exibe os tempos para leitura dos dados no HDFS. Com o posicionamento inicial dos blocos seguindo a PPR, a média dos tempos foi de 778,71s. Após o balanceamento, esse valor foi reduzido para 584,61s. Considerando a variação percentual dada por $\left(\left(T_{b}-T_{a}\right) / T_{a} \times 100\right)$, onde $T_{a}$ e $T_{b}$ equivalem, respectivamente, às médias aritméticas dos tempos das 20 execuções do benchmark TestDFSIO antes e após a execução do HDFS Balancer com a prioridade de confiabilidade dos racks, a variação alcançada foi de $-24,92 \%$, indicando a redução obtida no tempo de leitura dos dados.

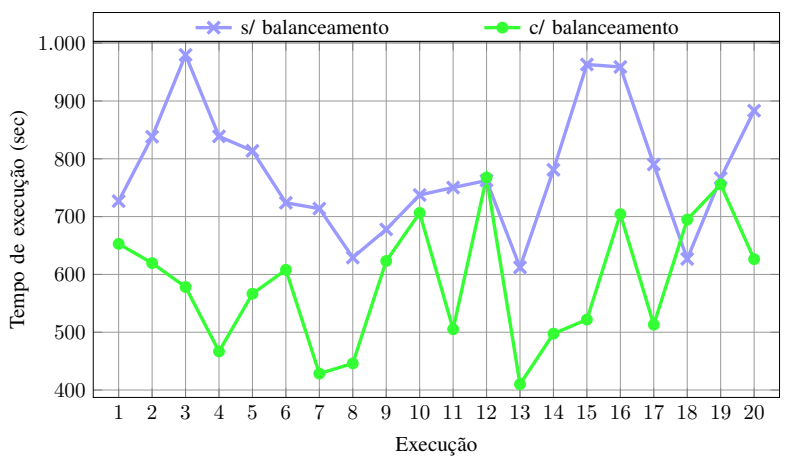

Figura 4. Tempo para leitura dos dados antes e após o balanceamento.

Com a capacidade do HDFS em processar conjuntos de tarefas paralelas e independentes sendo melhor explorada em um cluster equilibrado, outras métricas tornam-se passíveis 
de otimização. Na Figura 5, são exibidas as taxas de E/S alcançadas pelo benchmark. Essa taxa relaciona a velocidade de transferência média obtida por cada tarefa pela quantidade total de tarefas map executadas. Para o TestDF S IO, a quantidade de tarefas de mapeamento geradas equivale, por padrão, à quantidade de arquivos manipulados (no experimento, a leitura foi realizada sobre 10 arquivos). A taxa média de E/S foi de $47,33 \mathrm{MB} / \mathrm{s}$ sem o balanceamento e de $57,82 \mathrm{MB} / \mathrm{s}$ após o balanceamento com base na confiança dos racks. A variação percentual, considerando os valores médios das 20 execuções, foi de $22,16 \%$, indicando aumento na taxa de transferência.

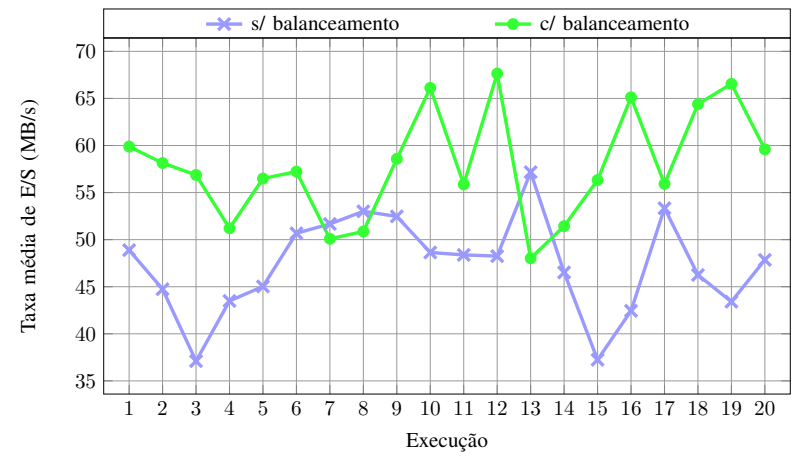

Figura 5. Taxa de transferência de dados antes e após o balanceamento.

A Figura 6 por sua vez, exibe o throughput obtido durante a leitura dos dados antes e após o equilíbrio do cluster. O throughput é dado pela razão do volume total de dados processados (em MB) pela soma dos tempos (em segundos) gastos por cada tarefa (devido ao paralelismo, este valor é superior ao tempo total de execução). O throughput médio foi de $45,7 \mathrm{MB} / \mathrm{s}$ sem o balanceamento e de $57,28 \mathrm{MB} / \mathrm{s}$ com o uso do balanceador. A variação percentual alcançada foi de $25,36 \%$, indicando aumento no througput da aplicação após o balanceamento com a prioridade de confiabilidade dos racks.

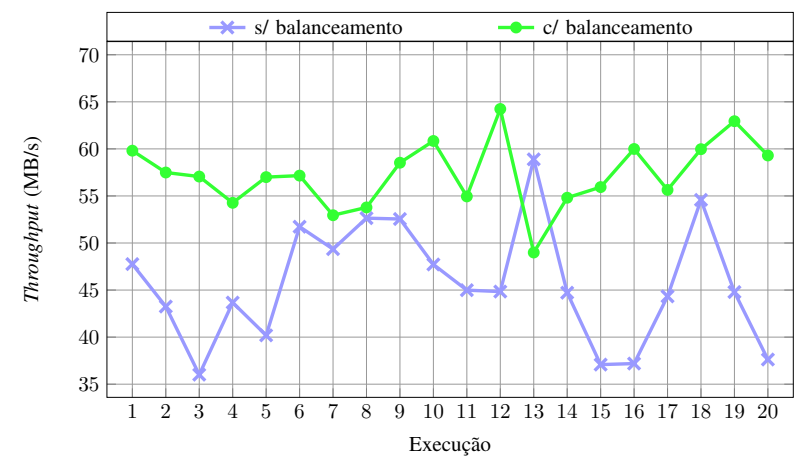

Figura 6. Throughput de leitura antes e após o balanceamento.

\section{CONSIDERAÇÕES FINAIS}

O HDFS Balancer é uma solução nativa do Hadoop voltada ao balanceamento de réplicas. Por operar de forma generalizada, sua política de balanceamento atual não considera possíveis demandas de disponibilidade e confiabilidade durante a redistribuição dos blocos entre os racks do cluster.
Esse trabalho apresentou uma estratégia de balanceamento customizada para o HDFS Balancer que prioriza racks com alta confiabilidade durante a movimentação das réplicas. Para tal, o fator de confiança de cada rack foi estimado com base na taxa de falhas de seus DataNodes. Após detalhar a implementação, conduziu-se uma investigação experimental que - além de validar a proposta - permitiu demonstrar que a execução do balanceador priorizando racks com menor ocorrência de falhas continua a proporcionar melhorias de desempenho significativas após o balanceamento de réplicas.

Trabalhos futuros envolvem avaliar a solução proposta em cenários com indução de falhas transientes em nodos e com testes de estresse considerando falha total de rack. Além disso, pretende-se associar a prioridade de confiabilidade dos racks apresentada neste trabalho com prioridades voltadas à otimização da disponibilidade dos dados no HDFS.

\section{AGRADECIMENTOS}

Os experimentos apresentados neste trabalho foram conduzidos na plataforma Grid'5000, apoiada por um grupo de interesses científicos hospedado por Inria e incluindo CNRS, RENATER e diversas Universidades, bem como outras organizações (mais detalhes em https://www.grid5000.fr).

\section{REFERÊNCIAS}

[1] Apache Software Foundation. (2018) Apache hadoop. [Online]. Available: https://hadoop.apache.org/docs/r2.9.2/ [Acesso: 19 de Maio, 2019].

[2] T. White, Hadoop: The Definitive Guide, 4th ed. Sebastopol: O'Reilly Media, Inc., 2015.

[3] R. W. A. Fazul and P. P. Barcelos, "Efetividade da política de posicionamento de blocos no balanceamento de réplicas do hdfs," in Anais do XX Workshop de Testes e Tolerância a Falhas. SBC, 2019, pp. 79-92.

[4] K. Shvachko, H. Kuang, S. Radia, and R. Chansler, "The hadoop distributed file system," in Symposium on Mass Storage Systems and Technologies. Incline Village: IEEE, 2010, pp. 1-10.

[5] Z. Guo, G. Fox, and M. Zhou, "Investigation of data locality in mapreduce," in Proceedings of the 2012 12th IEEE/ACM International Symposium on Cluster, Cloud and Grid Computing (ccgrid 2012). Ottawa: IEEE Computer Society, 2012, pp. 419-426.

[6] R. W. A. Fazul, P. V. Cardoso, and P. P. Barcelos, "Análise do impacto da replicação de dados implementada pelo apache hadoop no balanceamento de carga," in Anais do X Computer on the Beach. Florianópolis: Universidade do Vale do Itajaí, 2019, pp. 579-588.

[7] C. B. VishnuVardhan and P. K. Baruah, "Improving the performance of heterogeneous hadoop cluster," in Fourth International Conference on Parallel, Distributed and Grid Computing. IEEE, 2016, pp. 225-230.

[8] J. Dharanipragada, S. Padala, B. Kammili, and V. Kumar, "Tula: A disk latency aware balancing and block placement strategy for hadoop," in International Conference on Big Data. IEEE, 2017, pp. 2853-2858.

[9] K. Liu, G. Xu, and J. Yuan, "An improved hadoop data load balancing algorithm," Journal of Networks, vol. 8, no. 12, pp. 2816-2822, 2013.

[10] A. Shah and M. Padole, "Load balancing through block rearrangement policy for hadoop heterogeneous cluster," in 2018 International Conference on Advances in Computing, Communications and Informatics (ICACCI). Bangalore: IEEE, 2018, pp. 230-236.

[11] Hortonworks Data Plataform. (2018) Scaling namespaces and optimizing data storage. [Online]. Available: https://docs.hortonworks. com/HDPDocuments/HDP3/HDP-3.1.0/data-storage/content/balancing_ data_across_hdfs_cluster.html [Acesso: 2 de Junho, 2019].

[12] R. W. A. Fazul and P. P. Barcelos, "Política customizada de balanceamento de réplicas para o hdfs balancer do apache hadoop," in Anais do XX Workshop de Testes e Tolerância a Falhas. SBC, 2019, pp. 93-106.

[13] R. W. A. Fazul. (2019) Implementação de uma política customizada de balanceamento de réplicas para o hdfs balancer do apache hadoop. [Online]. Disponível em: https://repositorio.ufsm.br// Universidade Federal de Santa Maria, Centro de Tecnologia. (no prelo). 